\title{
Children and young adults hospitalized for severe COVID-19 exhibit thrombotic coagulopathy
}

\author{
William Mitchell ${ }^{1}$, Jennifer Davila ${ }^{1}$, Janine Keenan ${ }^{1}$, Jenai Jackson ${ }^{2}$, Adit Tal ${ }^{1}$, Kerry \\ Morrone $^{1}$, Ellen Silver ${ }^{2}$, Sarah O'Brien ${ }^{3}$, and Deepa Manwani ${ }^{1}$ \\ ${ }^{1}$ Children's Hospital at Montefiore \\ ${ }^{2}$ Yeshiva University Albert Einstein College of Medicine \\ ${ }^{3}$ Research Institute at Nationwide Children's Hospital
}

February 9, 2021

\begin{abstract}
We report the clinical and laboratory coagulation characteristics of 27 pediatric and young adult patients $(2$ months to 21 years) treated for symptomatic COVID-19 at a children's hospital in the Bronx, New York between March 1 and May 31, 2020. D-Dimer was > $0.5 \mathrm{ug} / \mathrm{mL}$ (upper limit of normal) in 25 (93\%) patients at admission; 11 (41\%) developed peak D-Dimer $>5 \mathrm{ug} / \mathrm{mL}$ during admission. Seven $(26 \%)$ patients developed venous thromboembolism: three with deep vein thrombosis and four with pulmonary embolism. Requirement of increased ventilatory support was a risk factor for thrombosis $(\mathrm{p}=0.006)$. Three of eight $(38 \%)$ patients on prophylactic anticoagulation developed thrombosis, however no patients developed VTE on low molecular weight heparin prophylaxis titrated to anti-Xa level. Manifestation of COVID-19 disease was severe or critical in $16(59 \%)$ patients. Four (15\%) patients died of COVID-19 complications: all had comorbidities. Elevated D-dimer and increased VTE rate were observed in this young cohort, particularly in those with severe respiratory complications suggesting thrombotic coagulopathy. More data is needed to guide thromboprophylaxis in this age group.
\end{abstract}

\section{Introduction}

Adult patients with severe COVID-19 develop a thrombotic coagulopathy, and numerous series have reported high rates of venous thromboembolism (VTE), including deep vein thrombosis (DVT) and pulmonary embolism (PE) ${ }^{1-4}$ however, these adult cohorts have median ages of 63-64 years. Data have been presented on pediatric cohorts with COVID-19, however, the associated coagulopathy has not been well described. ${ }^{5-10}$ In particular, whether an aggressive anticoagulation strategy, as has been implemented in some medical centers, is necessary, effective or safe for children and young adults is not clear. Defining the clinical characteristics and severity of the coagulopathy of COVID-19 in hospitalized pediatric patients is an urgent need. In this context, we performed a single-site retrospective review of 27 patients ages 2 months to 21 years, with confirmed, symptomatic COVID-19, admitted to a single Children's Hospital in the Bronx, New York. We describe the coagulopathy associated with COVID-19 in these hospitalized patients and how these data informed the iterative process that led to the development of institutional anticoagulation guidelines.

\section{Methods}

Institutional Review Board (IRB) of Montefiore Medical Center approval was obtained for this study. In this retrospective analysis, we present data on 27 patients admitted to the hospital for symptomatic COVID-19 between March 1 and May 31, 2020. Due to the emerging data on coagulopathy, all COVID-19 patients were followed by the Pediatric Hematology consult service during this time. Primary provider teams consulted the hematology service for all confirmed COVID-19 cases, which minimized selection bias. Only patients hospitalized for symptomatic COVID-19 were included: those admitted for another reason and found to 
have incidental COVID-19 infection were excluded. Diagnosis of COVID-19 was confirmed in all cases by nasopharyngeal swab PCR. D-dimer was measured using the Stago Liquid Xa assay in our clinical hematology laboratory.

Hospital records were reviewed for laboratory, clinical, radiographic and demographic data. This included comorbid conditions and concurrent medications. Descriptive statistics, including proportions, means, and standard deviations, were calculated for study variables. Associations of risk factors with thrombosis were analyzed using Chi-square or Fisher Exact tests and odds ratios (ORs) also were calculated for these comparisons. Additionally, a logistic regression analysis including the variables found to be significant in bivariate analyses was run to control for confounding and determine which factors independently predicted thrombosis. For each analysis any cases with missing data were excluded.

\section{Results}

This cohort of 27 children and young adults hospitalized with symptomatic COVID-19 (Table 1) was roughly equal by gender, with $14(52 \%)$ males and $13(48 \%)$ females. The numbers of patients in different age ranges were $3(11 \%)$ less than 1 year, 4 (15\%) 1 through 6 years, 8 (30\%) 7 through 12 years, and $12(44 \%) 13$ through 21 years (Table 1). The background racial and ethnic makeup of the Bronx, New York based on the 2019 census report is approximately $44 \%$ Black and $45 \%$ White, with $56 \%$ of all races identifying as Hispanic or Latino ethnicity. In this cohort 3 (11\%) patients self-identified as Black, 1 (4\%) White, $11(41 \%)$ other, and 16 (59\%) patients self-identified as Spanish/Hispanic/Latino. Obesity and sickle cell disease were the most prevalent comorbid conditions, with $5(19 \%)$ patients having BMI $>95^{\text {th }}$ percentile, and $5(19 \%)$ having sickle cell anemia.

Clinically, 16 (59\%) patients in this cohort had severe or critical illness due to COVID-19, based on established criteria (grades asymptomatic, mild, moderate, severe, critical) ${ }^{6}$, and corresponding to grades 5 and higher by the WHO progression scale (grades 1-10), ${ }^{11}$ and 14 (52\%) patients required increased ventilatory support. This was defined by the need for $>5 \mathrm{~L}$ oxygen via nasal cannula, high-flow nasal cannula, nonrebreather mask, or intubation. These patients were labelled as the "High Ventilatory Support" sub-group. Patients not requiring any ventilatory support or only oxygen supplementation of $<5 \mathrm{~L}$ oxygen via nasal cannula were labeled as the "Low Ventilatory Support" sub-group. $9(33 \%)$ patients required intubation. Four (15\%) patients died of COVID-19 complications.

Coagulation parameters were abnormal in much of the cohort (Table 1). Only one (5\%) of the 22 patients whose D-dimer was measured had a D-Dimer $<0.5 \mathrm{ug} / \mathrm{mL}$ (institutional normal range $0.27-0.50 \mathrm{ug} / \mathrm{mL}$ ) and the mean peak D-dimer value was $6.41 \mathrm{ug} / \mathrm{mL}$ (range $0.27-16.9 \mathrm{ug} / \mathrm{mL}$ ). PT and PTT were prolonged with mean peak values of 17.8 seconds (range 13.8-43.3 seconds) and 65.6 seconds (range 29.3 - 200 seconds), respectively (ULN 14.8 seconds and 44.8 seconds, respectively). Platelet counts were primarily in the normal or high range, with mean peak count of $416 \mathrm{k} / \mathrm{uL}$ (range $183-789 \mathrm{k} / \mathrm{uL}$ ). There were $11(41 \%)$ patients with platelets $<150 \mathrm{k} / \mathrm{uL}$ (institutional lower limit of normal), and the mean platelet nadir of the cohort was $175 \mathrm{k} / \mathrm{uL}$ (range $5-683 \mathrm{k} / \mathrm{uL}$ ). Seven $(26 \%)$ patients were significantly thrombocytopenic $(<50 \mathrm{k} / \mathrm{uL})$. Fibrinogen was elevated with mean peak value of $642 \mathrm{mg} / \mathrm{dL}$ (range $227-1800 \mathrm{mg} / \mathrm{dL}$, ULN $283 \mathrm{mg} / \mathrm{dL}$ ). All lab testing was performed in the hospital laboratory with the usual quality assurance measures in place.

Venous thromboembolism (VTE) was identified in 7 (26\%) patients (Table 2). Three (11\%) patients developed DVT and 4(15\%) developed PE. One of these patients had bilateral PE. A requirement for increased ventilatory support was a risk factor for VTE, with 7 of $14(50 \%)$ patients in the High Ventilatory Support group developing VTE as compared to none of 13 patients in the Low Ventilatory Support group $(\mathrm{p}=0.006)$ (Table 3). Elevated D-dimer was not a statistically significant risk factor for VTE in the cohort, potentially due to small sample size. However, there was a trend of D-dimer $>5 \mathrm{ng} / \mathrm{uL}$ in patients with 
VTE, with 2 of $11(18 \%)$ and 5 of $11(45 \%)$ patients developing VTE with D-dimer $<5$ and $>5 \mathrm{ug} / \mathrm{mL}$, respectively. Obesity was not associated with VTE in our cohort, potentially due to inadequate statistical power from a small sample size. All three patients with DVT and 2 of $4(50 \%)$ patients with PE (total 5 of $7,71 \%)$ had central venous lines, and 11 of $20(59 \%)$ without VTE had central venous lines. This difference too did not reach statistical significance $(\mathrm{p}=.446)$, perhaps due to small sample size. Given our relatively small sample size and the correlated nature of these variables, the independent contribution of each risk factor to VTE could not be statistically analyzed by regression modeling.

Children and young adults with symptomatic COVID-19 were at risk for VTE despite prophylactic anticoagulation, as $4(57 \%)$ of the 7 patients with confirmed VTE were on prophylaxis. Eleven (41\%) patients received prophylactic anticoagulation, with $10(91 \%)$ of those patients receiving low molecular weight heparin (LMWH, enoxaparin) and 1 (9\%) 20 year old patient receiving apixaban. The LMWH was dosed at 0.75 $\mathrm{mg} / \mathrm{kg}$ BID for neonates up to 2 months, and $0.5 \mathrm{mg} / \mathrm{kg}$ BID for over 2 months, with titration of anti-Xa level to $0.2-0.4 \mathrm{ng} / \mathrm{mL}$ (Based on CHEST guidelines ${ }^{12}$ and institutional anticoagulation guidelines).

Six $(60 \%)$ of the 10 patients receiving LMWH had their dose titrated to ant-Xa level, and none of these patients developed VTE. Four patients received fixed dose LMWH, which was either $40 \mathrm{mg}$ per day (standard adult prophylactic dose) or fixed dosing based on weight (generally for obese patients ${ }^{13}$ ). Three $(75 \%)$ of the 4 patients receiving fixed-dose prophylactic LMWH developed VTE, of these individuals one was obese. The remaining patient on prophylaxis was on apixaban, $2.5 \mathrm{mg}$ twice daily, and developed a PE. Although the numbers are small, we did not see breakthrough VTE on anti-Xa-monitored prophylactic dosing of LMWH. No clear pattern was identified in the subset that developed VTEs on prophylactic anticoagulation, except the for the anticoagulation choice/regimen. The remaining 3 confirmed VTEs occurred in patients on therapeutic dosing of anticoagulation. Since therapeutic anticoagulation was often initiated in the most ill patients, comparison of VTE rates to the patients on prophylaxis is not feasible. It should be noted that all patients in this cohort were started on guideline based anticoagulation upon admission. Thus, unless their VTE developed before admission, these patients all received anticoagulation before VTE diagnosis and delayed start of anticoagulation was not identified as the cause for breakthrough VTE. Overall, the anticoagulation was well tolerated and no cases of grade 4 or higher bleeding were observed. Two patients had oozing from indwelling central venous line insertion sites and one of those had hematuria but neither had life-threatening hemorrhage.

\section{Discussion}

During the 2020 COVID-19 surge in New York City, there were over 200,000 confirmed cases in New York City, with almost 17,000 confirmed deaths by May 31 ${ }^{\text {st }}$,2020. (https://www1.nyc.gov/). Almost all data emerging from this time is restricted to the older adult population with average of age of cohorts ranging between $63-65$ years $^{1,14,15}$. There has been limited information on the impact of COVID-19 on the coagulation of pediatric and young adult patients. Herein we present data revealing that children and young adults with symptomatic COVID-19 do in fact suffer from the severe coagulopathy that has been reported in adult patients and can become severely or critically ill. Those patients who died were 2 months, 11,14 and 18 years old, and all had comorbidities, which were cardiac defect, cancer, genetic syndrome and trisomy 21, respectively. One of these patients also developed a DVT. Comorbidities may also have contributed to risk for COVID-19 related VTE in this cohort. Although obesity, central venous line and sickle cell were not risk factors for VTE in this cohort, there was a predominance of Black and Hispanic patients. In the US, there are known higher rates of infection, morbidity, and mortality in these populations. ${ }^{16,17}$

Contrary to expectations, sickle cell disease was not associated with an increased risk of thrombosis: none of 5 patients developed VTE (Supplemental Table S1). SCD and non-SCD patients did not differ in baseline 
demographic and clinical characteristics, except for the expected preponderance of patients who self-identified as Black in the SCD group (data not shown). Although all 5 patients with SCD were admitted with acute chest syndrome, none developed severe or critical illness or required high ventilatory support, and none had D-dimer $>5 \mathrm{mcg} / \mathrm{mL}$. Two of the SCD patients were on chronic transfusions and none were on hydroxyurea. The clinical course of these patients is described in greater detail in a prior publication from our group ${ }^{18}$.

A limitation of this study is that it included a relatively small number of patients from one medical center, which may have introduced treatment bias. Importantly, this study included only those patients admitted for symptomatic COVID-19. Patients found to have incidental, asymptomatic COVID-19 were excluded. Laboratory evaluation of this novel disease evolved over time as the pandemic continued. In particular, D-dimer was not reported for about $25 \%$ of the earliest patients in the cohort, which may have affected the statistical association of this test with VTE. While the odds ratios of D-dimer and central venous line as risk factors were fairly high, they failed to reach significance due to small numbers. An association of D-dimer elevation with mortality and thrombosis has been observed in adult cohorts and deserves further study in future pediatric cohorts with larger sample size. ${ }^{20,21}$ Peak values of the activated partial thromboplastin time (aPTT) were noted to be significantly elevated in this cohort which is different from what has been reported in adult cohorts. However, the aPTT standard deviation was large due to 4 outlier values for which heparin contamination could not be ruled out. Additionally, the presence of a lupus anticoagulant was not tested for in this population. Even with these 4 values removed the mean peak aPTT remained prolonged (mean 48.59s, SD 6.19s, ULN 43.8s) while the mean PTT at admission was in the normal range (mean 35.5s, SD 6.2s). These hemostasis variables will need to be explored in a larger sample size to determine their exact impact on VTE in pediatric and young adult patients with COVID-19.

In the course of the pandemic it was difficult logistically to obtain imaging with either sonography or CT scan for patients with COVID-19 due to isolation precautions. This limited our ability to determine the absolute incidence of VTE. As our guidelines evolved, imaging was recommended for all patients but was still not uniformly available. This potentially limited our ability to diagnose VTE on this cohort. Of those 16 who did have imaging, 7 (43\%) had VTE. Nine patients had extremity dopplers, $2(22 \%)$ of whom had VTE and both were symptomatic. No asymptomatic line related clots were diagnosed, although the entire cohort was not screened. One patient with incidental portal vein thrombosis was asymptomatic for VTE but had an abdominal sonogram for abnormal LFTs. Four (57\%) of the 7 patients imaged for PE were positive - all were symptomatic. However, imaging was limited due to logistics of COVID-19-related restrictions and not all patients symptomatic for possible PE had imaging in real time. We did not observe any stroke in this cohort and did not perform any head imaging to assess for stroke.

Anticoagulation guidelines were put in place initially based on the data from Wuhan reporting decreased mortality with heparin use ${ }^{15}$, as well as our early findings of VTE in untreated children and young adults. The youngest patient in our cohort to have a VTE was two months old, leading us to recommend prophylactic anticoagulation for pediatric patients of all ages hospitalized with symptomatic COVID-19. One patient presented with PE ten days post discharge, leading us to recommend prophylactic anticoagulation for two weeks post discharge. This patient presented with pneumonia and required high flow nasal cannula on both admissions. We used the evolving data from our patients to inform an iterative weekly analysis by which to base our institutional anticoagulation guidelines. In the absence of bleeding risk, we recommended antiXa-monitored LMWH prophylaxis, with pre-emptive escalation to therapeutic dosing in patients requiring high ventilatory support or with D-dimer levels $>5 \mathrm{ug} / \mathrm{mL}$. An increased rate of breakthrough thrombosis despite prophylactic anticoagulation has been reported in severely ill adult patients ${ }^{14}{ }^{19}$, highlighting the need for further evaluation of effective regimens in clinical trials. The use of D-dimer as a risk factor for thrombosis in COVID-19 was based on adult literature, as no pediatric data existed at that time. ${ }^{20,21}$ The 
D-dimer cutoff of 10x our institutional ULN was based both on the observation that patients developing VTE generally had D-dimer $>5 \mathrm{u} / \mathrm{mL}$ and the desire to limit use of higher doses of anticoagulation. Doses and monitoring were different for patients with obesity ${ }^{13}$ or acute kidney injury ${ }^{22}$. In patients with renal dysfunction LMWH was used once per day with monitoring of anti-Xa peak and trough levels to prevent accumulation.

In repeated data analysis there was evidence of a clear demarcation between those patients who needed ventilatory support of $<5$ liters nasal cannula, and those requiring more aggressive ventilatory management with high-flow nasal cannula, non-rebreather mask or intubation. Of note, this level of ventilatory support is typically criteria for admission to our intensive care unit (ICU). However, because of high ICU volume, primarily only intubated patients were admitted to the ICU during this period. The rates for confirmed VTE were 0 and $50 \%$ in the low and high ventilatory support cohorts, respectively. Based on this data and the limited availability of imaging, we opted to treat patients requiring $>5 \mathrm{~L} \mathrm{NC}$ ventilatory support with full dose anticoagulation with LMWH, titrated to an anti-Xa level of $0.6-1.1 \mathrm{ng} / \mathrm{mL}$, which was well tolerated in this age group. Subsequent to the data collection and reporting period, both the American Society of Hematology and the International Society of Thrombosis and Haemostasis published guidance on anticoagulation in adults with COVID-1923, 24 . Based on these publications and our own findings, we further revised our guidelines to uniformly image patients requiring high ventilatory support or with D-dimer levels $>5 \mathrm{ug} / \mathrm{mL}$, rather than preemptively escalating to therapeutic anticoagulation (Supplemental Figure S1). It must be pointed out that these institutional guidelines for thromboprophylaxis management evolved as the cohort accrued and as more adult data became available. Their use is subject to change based on the evolving body of literature. Ongoing and future studies will determine if intensified thromboprophylaxis, therapeutic dosing anticoagulation, addition of antiplatelet or anti-inflammatory agents are the correct approach to address the severe COVID-19 coagulopathy in both children and adults. Ongoing clinical trials may soon answer these questions in adults (the ACTIV-4 studies, NCT04505774 and NCT04498273).

In conclusion, while many children and young adults may not require admission to the hospital for COVID19 , the laboratory picture and clinical course of more severely ill children and young adults is similar to that of older adult patients. Coagulopathy, elevated D-dimer and increased VTE rate were observed in this young cohort, particularly in those with severe respiratory complications. More data is needed to guide thromboprophylaxis in this age group. However, until more data from larger studies is available, care will continue to be guided by the adult experience. In this time of limited experience with this novel infection, we have closely observed our patients to guide us in how best to care for them. We hope our findings will inform future management of children and young adults hospitalized with symptomatic COVID-19.

Authorship: WBM collected and analyzed data and wrote the manuscript, JJ collected data, JD collected data and revised the manuscript, AT collected data and revised the manuscript, KAM revised the manuscript, EJS performed statistical analyses, SOB analyzed data and revised the manuscript, DM oversaw data collection, analyzed data and revised the manuscript

Conflict of interest statement: The authors declare no competing financial conflicts.

\section{References}

1. Helms J, Tacquard C, Severac F, et al. High risk of thrombosis in patients with severe SARS-CoV-2 infection: a multicenter prospective cohort study. Intensive Care Med. May 4 2020:1-10. doi:10.1007/s00134020-06062-x

2. Iba T, Levy JH, Levi M, Connors JM, Thachil J. Coagulopathy of Coronavirus Disease 2019. Crit Care Med. May 27 2020;doi:10.1097/ccm.0000000000004458 
3. Iba T, Levy JH, Levi M, Thachil J. Coagulopathy in COVID-19. J Thromb Haemost. Sep 2020;18(9):2103-2109. doi:10.1111/jth.14975

4. Iba T, Connors JM, Levy JH. The coagulopathy, endotheliopathy, and vasculitis of COVID-19. Inflamm Res. Dec 2020;69(12):1181-1189. doi:10.1007/s00011-020-01401-6

5. Castagnoli R, Votto M, Licari A, et al. Severe Acute Respiratory Syndrome Coronavirus 2 (SARS-CoV-2) Infection in Children and Adolescents: A Systematic Review. JAMA Pediatrics. 2020;doi:10.1001/jamapediatrics.2020.1467

6. Dong Y, Mo X, Hu Y, et al. Epidemiology of COVID-19 Among Children in China. Pediatrics. 06 2020;145(6)doi:10.1542/peds.2020-0702

7. Du H, Dong X, Zhang JJ, et al. Clinical characteristics of 182 pediatric COVID-19 patients with different severities and allergic status. Allergy. Jun 2020;doi:10.1111/all.14452

8. Romani L, Chiurchiù S, Santilli V, et al. COVID-19 in Italian pediatric patients: the experience of a tertiary children's hospital. Acta Paediatr. Jul 2020;doi:10.1111/apa.15465

9. Jiang L, Tang K, Levin M, et al. COVID-19 and multisystem inflammatory syndrome in children and adolescents. Lancet Infect Dis. Nov 2020;20(11):e276-e288. doi:10.1016/s1473-3099(20)30651-4

10. Shekerdemian LS, Mahmood NR, Wolfe KK, et al. Characteristics and Outcomes of Children With Coronavirus Disease 2019 (COVID-19) Infection Admitted to US and Canadian Pediatric Intensive Care Units. JAMA Pediatr. Sep 1 2020;174(9):868-873. doi:10.1001/jamapediatrics.2020.1948

11. A minimal common outcome measure set for COVID-19 clinical research. Lancet Infect Dis. Aug 2020;20(8):e192-e197. doi:10.1016/s1473-3099(20)30483-7

12. Monagle P, Chan AKC, Goldenberg NA, et al. Antithrombotic therapy in neonates and children: Antithrombotic Therapy and Prevention of Thrombosis, 9th ed: American College of Chest Physicians EvidenceBased Clinical Practice Guidelines. Chest. Feb 2012;141(2 Suppl):e737S-e801S. doi:10.1378/chest.11-2308

13. Wang TF, Milligan PE, Wong CA, Deal EN, Thoelke MS, Gage BF. Efficacy and safety of high-dose thromboprophylaxis in morbidly obese inpatients. Thromb Haemost. Jan 2014;111(1):88-93. doi:10.1160/th13-01-0042

14. Klok FA, Kruip M, van der Meer NJM, et al. Incidence of thrombotic complications in critically ill ICU patients with COVID-19. Thromb Res. Apr 10 2020;doi:10.1016/j.thromres.2020.04.013

15. Tang N, Bai H, Chen X, Gong J, Li D, Sun Z. Anticoagulant treatment is associated with decreased mortality in severe coronavirus disease 2019 patients with coagulopathy. J Thromb Haemost. May 2020;18(5):1094-1099. doi:10.1111/jth.14817

16. Pan D, Sze S, Minhas JS, et al. The impact of ethnicity on clinical outcomes in COVID-19: A systematic review. EClinicalMedicine. Jun 2020;23:100404. doi:10.1016/j.eclinm.2020.100404

17. Rentsch CT, Kidwai-Khan F, Tate JP, et al. Covid-19 by Race and Ethnicity: A National Cohort Study of 6 Million United States Veterans. medRxiv. May 2020;doi:10.1101/2020.05.12.20099135

18. Morrone KA, Strumph K, Liszewski MC, et al. Acute chest syndrome in the setting of SARSCOV-2 infections-A case series at an urban medical center in the Bronx. Pediatric Blood $\mathscr{G}$ Cancer. 2020;67(11):e28579. doi:10.1002/pbc.28579

19. Llitjos JF, Leclerc M, Chochois C, et al. High incidence of venous thromboembolic events in anticoagulated severe COVID-19 patients. J Thromb Haemost. Apr 22 2020;doi:10.1111/jth.14869

20. Avruscio G, Camporese G, Campello E, et al. COVID-19 and Venous Thromboembolism in Intensive Care or Medical Ward. Clin Transl Sci. Nov 2020;13(6):1108-1114. doi:10.1111/cts.12907 
21. Sun Y, Dong Y, Wang L, et al. Characteristics and prognostic factors of disease severity in patients with COVID-19: The Beijing experience. J Autoimmun. Aug 2020;112:102473. doi:10.1016/j.jaut.2020.102473

22. Nutescu EA, Spinler SA, Wittkowsky A, Dager WE. Low-molecular-weight heparins in renal impairment and obesity: available evidence and clinical practice recommendations across medical and surgical settings. Ann Pharmacother. Jun 2009;43(6):1064-83. doi:10.1345/aph.1L194

23. Cuker A. ASH 2020 guidelines on the use of anticoagulation in patients with COVID-19: Draft recommendations.

24. Goldenberg NA, Sochet A, Albisetti M, et al. Consensus-based clinical recommendations and research priorities for anticoagulant thromboprophylaxis in children hospitalized for COVID-19-related illness. $J$ Thromb Haemost. Nov 2020;18(11):3099-3105. doi:10.1111/jth.15073

\section{Hosted file}

Table 1 R2.docx available at https://authorea.com/users/363074/articles/483985-children-andyoung-adults-hospitalized-for-severe-covid-19-exhibit-thrombotic-coagulopathy

\section{Hosted file}

Table 2 R2.docx available at https://authorea.com/users/363074/articles/483985-children-andyoung-adults-hospitalized-for-severe-covid-19-exhibit-thrombotic-coagulopathy

\section{Hosted file}

Table 3.docx available at https://authorea.com/users/363074/articles/483985-children-andyoung-adults-hospitalized-for-severe-covid-19-exhibit-thrombotic-coagulopathy

\section{Hosted file}

SupTable S1 R2.docx available at https://authorea.com/users/363074/articles/483985-childrenand-young-adults-hospitalized-for-severe-covid-19-exhibit-thrombotic-coagulopathy

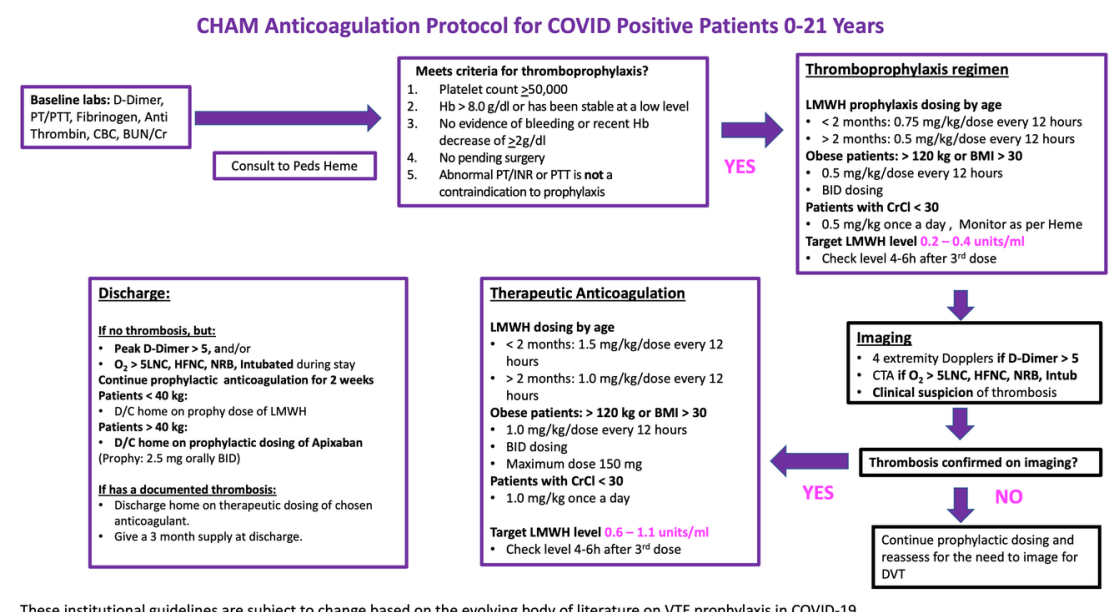

Figure 1: This is a caption 